\title{
DETECTING GRAVITATIONAL WAVES WITH PULSARS
}

\author{
Daniel R. Stinebring
}

Physics \& Astronomy Dept., Oberlin College, Oberlin, OH 44074, USA

Ein instein's theory of General Relativity predicts that accelerating masses will produce gravitational radiation: ripples in space-time that propagate outward at the speed of light and carry energy away from the source. The effect of this energy loss in the first-discovered double neutron star system was unambiguously detected by Joseph Taylor, Russell Hulse, and collaborators in the 1980's and garnered the Nobel prize in physics for them in 1993. But, the direct detection of gravitational waves (GW) from cosmic sources remains one of the great quests of modern physics, and groups around the world are using a variety of techniques in the search.

For example, detectors such as the European-based VIRGO and GEO600 and the US-based LIGO use powerful lasers directed down orthogonal arms several kilometers long to attempt to detect the passage of a GW by sophisticated extensions of Michelson interferometry [1]. These detectors are sensitive to gravitational waves with periods comparable to the light travel time across the array: tens of microseconds. Various techniques to lengthen the response time plus the fact that many sources of gravitational waves are expected to rise in spectral power at lower frequencies produces a peak sensitivity around $0.1 \mathrm{~s}$ or $\mathrm{f} \sim 10 \mathrm{~Hz}$. A proposed space-based array, LISA, the Laser Interferometer Space Antenna, based on the same Michelson interferometer principle but with lasers directed between free-flying stations $5 \times 10^{6} \mathrm{~km}$ apart in a one year orbit around the Sun, would have a peak sensitivity around $\mathrm{f} \sim 10^{-3} \mathrm{~Hz}$. (A precursor LISA Pathfinder mission is an approved ESA project with an expected launch in 2009.)

\section{Pulsar timing - a complementary technique}

Pulsars - rotating, highly magnetized neutron stars - are themselves the product of dramatic gravitational collapse of massive stars and provide another means to detect gravitational waves directly. The technique uses electromagnetic radiation from a group of pulsars to search for GWs, but the technique is not interferometric in character. Rather, radio astronomers search for slight disturbances in the arrival time of the pulses from these 'radio lighthouses' to signal the presence of gravitational waves. This technique is most sensitive to gravitational waves with frequencies about equal to the inverse length of the data record, which is tens of years in the best cases. This results in sensitivity to GWs in the nanohertz range, complementary to the much higher frequency range probed by the interferometric techniques.

The discovery in 1982 of a class of rapidly rotating yet very old $\left(\sim 10^{9} \mathrm{yr}\right)$ "millisecond pulsars," revolutionized the prospects for gravitational wave detection with this method. Millisecond (or recycled) pulsars have gone through an accretion-induced spin-up phase that leaves many of them with rotational periods less than $10 \mathrm{~ms}$. Not only do these pulsars have a timing precision $100-1000$ times better than the more numerous longer-period objects, but their relatively old ages and small spindown rates (i.e., slowing down of rotation) makes them less prone to the minor rotational irregularities that are present in younger pulsars.

The observations do not track the exact phase of the electromagnetic waves (as in Michelson interferometry) arriving from the pulsar at distance of hundreds to thousands of light years, but the rotational phase of the pulsar is measured instead. Since the width of the pulsar radio beam can be as narrow as $40 \mu \mathrm{s}$, high signal-to-noise ratio observations with the world's largest radio telescopes (Fig. 1) can determine the arrival phase of the pulse to a precision as good as $0.1-0.2 \mu \mathrm{s}$ in the best cases.

\section{Gravitational waves and pulsar timing}

What effect do cosmic gravitational waves have on this electromagnetic link? The passage of a gravitational wave past the pulsar at the time the radio waves are emitted causes an effective Doppler shift in the repetition frequency of the pulses of order $\left(\nu-v_{0}\right) / v_{0} \approx h$, where $h$ is the dimensionless strain that characterizes the GW. This shifts the observed pulse phase by a slight amount, $\delta t \approx h T$, where $T$ is the duration of the disturbance: weeks to years to decades in the cases of interest here. Similarly, the presence of a gravitational wave at the Earth at the time of reception of the e-m wave will introduce another slight time shift into the measurement. Surprisingly, the presence of gravitational waves along the hundreds to thousands of light year journey through the Milky Way galaxy does not affect the signal at all. This immunity to disturbances along the travel path arises because of two properties shared by electromagnetic waves and gravitational waves: they are both transverse, and they both travel at $c$ in a vacuum. This leads to a cancellation of effects along the propagation path, and even the slight changes in propagation speed of the e-m wave because interstellar space is a dilute plasma are not enough to produce a detectable GW signal due to metric variations along the path. So, it is simply the metric disturbance (value of $h$ ) at the pulsar at the time of emission and, similarly, of the Earth at the time of reception that determines the net value of $\delta t$ in the pulsar timing observation. There are, of course, additional complications (two independent polarizations of the $\mathrm{GW}$, geometrical factors, etc.), but this is the essential physics [2,3].
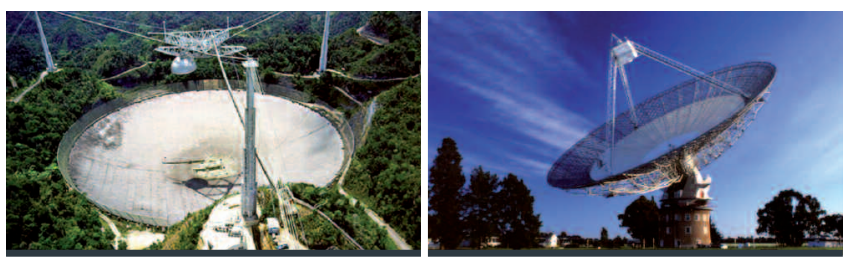

$\triangle$ FIG. 1: The Arecibo Observatory in Puerto Rico (left) and the Parkes Observatory in Australia (right) figure prominently in the search for gravitational waves using pulsars. Arecibo, the world's largest radio telescope, has unsurpassed sensitivity and discovered the first binary pulsar in 1974 and the first millisecond pulsar in 1982. The Parkes radio telescope can observe many of the millisecond pulsars that form the basis for a pulsar timing array (PTA) and is used in the best-developed PTA effort. 


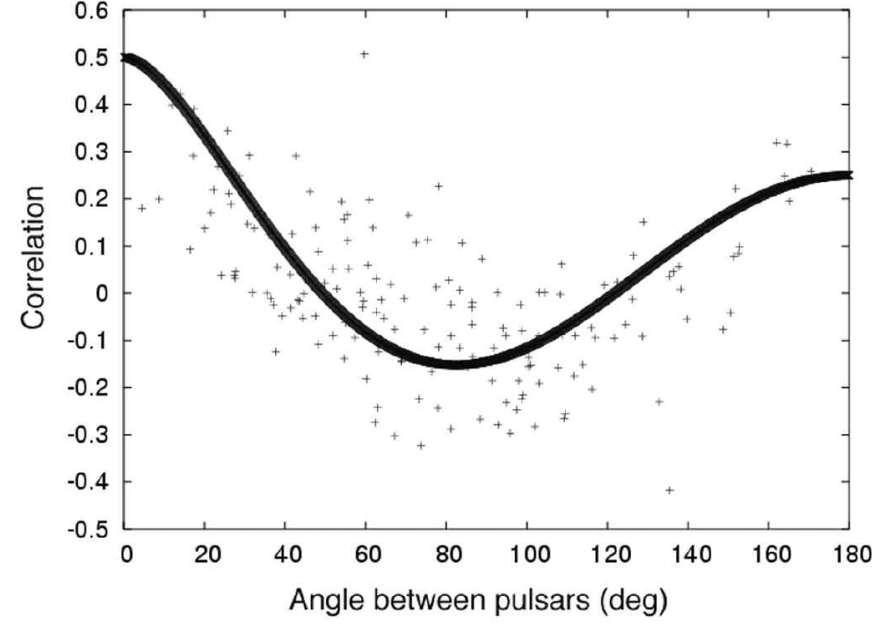

$\Delta$ FIG. 2: Half the timing irregularities due to a background of gravitational waves will be correlated around the sky because the gravitational waves are "jostling" the Earth, causing a retardation or advance of the observed pulsar rotational phase. The curved line in this plot shows the expected correlation as a function of angle between the pulsars. The plotted points are a simulation of the pair-wise timing correlations of 20 pulsars spread around the sky and represent one kind of signal that a PTA could detect [9].

There is one important caveat. Unlike the case of spectral lines in atomic physics, the undisturbed rotation frequency of the pulsar $\left(v_{0}\right)$ and its first derivative because of pulsar spindown are not known a priori. Hence, the arrival phase of the pulse must be fit by a model that includes terms linear and quadratic in the observation time, $t$. Other parameters of the system must also be fit for such as the exact position of the pulsar on the sky, its motion across the sky, and multiple parameters of a binary companion, if one exists - and many millisecond pulsars are in binary systems. This reduces the sensitivity of the data in ways that must be carefully modeled $[4,5]$.

How can this behaviour be used to detect gravitational waves with a pulsar timing array (PTA)? Consider a stochastic background of gravitational waves permeating the Galaxy. The effect on each of the pulsars being monitored will be independent since their GW-induced motion will be uncorrelated due to the large distances between them. However, half of the GW-induced timing noise in each pulsar-Earth link will be due to the effects of the GW on the Earth, and these effects will be correlated with a correlation coefficient that depends on the angular separation between the pulsars (Fig. 2) [3,6].

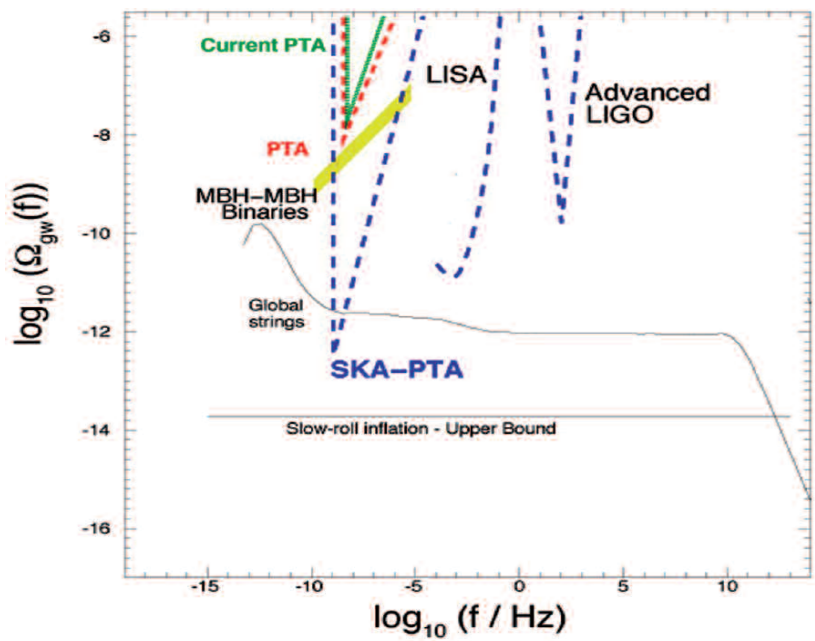

\section{Sources of nanohertz gravitational waves}

Take an electric charge and shake it back and forth. You will produce a kink in the electric field that will propagate away as an electromagnetic wave. The same would be true if you took a mass and shook it back and forth. Unfortunately from the standpoint of detecting gravitational waves, there are no hands out in the universe shaking massive objects back and forth. Instead, massive objects accelerate because of the presence of other massive objects. But, General Relativity postulates that, locally at least, all forms of mass-energy accelerate at the same rate. The static gravitational field itself, being a form of such mass-energy, then accelerates in the same fashion as the object that is producing it. Thus, massive, accelerating objects are very inefficient at introducing kinks into the field that surrounds them. It is only the non-local (i.e. tidal) aspects of the field that give rise to gravitational waves [7].

What might produce gravitational waves in the nanohertz frequency range? Collisions between galaxies and the ultimate coalescence of the massive black holes (MBH) at their centres are expected to emit strongly in this frequency range, but the signal from any one merging event is weak. However, the incoherent sum of thousands of such ongoing events form a stochastic background of gravitational waves that should be detectable by pulsar timing efforts in the next five to ten years $[8,9]$.

Early-universe phase transitions and the emergence of various topological defects should also produce a stochastic background of gravitational waves $[10,11]$. However, there is a much wider range of model predictions in the case of a primordial GW background than for a background of MBH binaries; hence, a particular observational limit on a GW background affords more "wiggle-room" for these models.

A periodic or chirped signal from a single orbital system can be characterized by the strain amplitude $h$ and the frequency of the wave $f$, both of which change with time during a gravitational coalescence event. However, for a stochastic background of gravitational waves it is conventional to express the spectral power as a fraction of the closure density of the universe. The fractional energy density per unit logarithmic frequency interval is $\Omega_{g u}(f)=\left(2 \pi^{2} / 3 H_{0}^{2}\right) f^{2} b^{2}$, where $H_{0}$ is the Hubble factor. A variety of possible sources of a GW background and the instrumental sensitivities, achieved and anticipated, are plotted in Fig. 3.

4FIG. 3: The power in a gravitational wave background as a function of GW frequency for a variety of sources relevant to PTAs. Power is shown as the ratio, per logarithmic frequency interval, of the stochastic gravitational wave power divided by the critical energy density of the universe. PTA refers to a generic pulsar timing array. Both current limits and five year projected improvements are shown. SKA-PTA is the pulsar timing array possible when the Square Kilometer Array is built in the 2015 2020 time frame. The most promising candidate for a stochastic GW source in this frequency range is due to massive black holes $\left(10^{7}-10^{8}\right.$ solar masses) that in-spiral and coalesce as galaxies collide (shown as a yellow bar). Stochastic backgrounds produced by cosmic strings or in an inflationary era are also shown. Projected sensitivity limits are shown for LISA and Advanced LIGO, but the astrophysical sources most relevant to those detectors, binary combinations of stellar mass black holes and neutron stars, are not indicated. [The original figure, modified here, is courtesy of G. Hobbs.] 


\section{Pulsar Timing Arrays: present and future}

There are several, partially overlapping, efforts underway to detect a GW background with pulsars. The Parkes Pulsar Timing Array (PPTA) is the oldest and most fully developed collaboration $[8,9]$. Their goal is to make multi-frequency timing observations of 20 of the brightest millisecond pulsars observable with the Parkes 64-m radio telescope on an approximately biweekly basis. This well-established observing and analysis program is publishing results on several scientific fronts related to the ultimate goal of detecting a GW background. The European Pulsar Timing Array (EPTA) takes advantage of three existing large-aperture telescopes: Jodrell Bank Observatory (UK), the Westerbork Synthesis Radio Telescope (the Netherlands), the Effelsberg Radio Telescope (Germany), and will be joined by the Sardinia Radio Telescope (Italy) when it is completed [12]. The North American collaboration, NANOGrav, uses data from the Arecibo Observatory, the largest radio telescope in the world, and the Byrd Green Bank Telescope [13]. All three collaborations share techniques, some data, and they have overlapping lists of personnel. Lamentably, both the Parkes Observatory and the Arecibo Observatory are dealing with severe budget cutbacks despite the crucial role they play in this forefront effort.

The longer-term future of pulsar-based gravitational wave searching will be focused on the Square Kilometer Array (Fig. 4), a world-wide effort with headquarters at the University of Manchester. When completed near the end of the next decade, this huge array of detectors/telescopes in a radio-quiet site in either Australia or southern Africa, will revolutionize all areas of radio astronomy. The discovery of an estimated 1000 or so millisecond pulsars and the ability to time 10 or 20 of them at a level of precision of $100 \mathrm{~ns}$ or better, will lead to a sensitivity level for this PTA of as much as a factor of $10^{4}$ over existing efforts $[14,15]$.

\section{Status of Results and Prospects}

As is evident in Fig. 3, pulsar timing is rapidly approaching a detection or falsification threshold for a GW background from coalescing massive black hole binaries. The latest upper bounds, using 8 years of data, place a limit of $\Omega_{g w} \leq 4 \times 10^{-8}$ on this source of a background and similar limits on that due to either relic gravitational waves from quantum era fluctuations or from the decay of cosmic strings [5].

The effort to use pulsars to detect individual GW sources or, more likely, a stochastic background of such sources is well underway using the world's largest radio telescopes. The nanohertz frequency range being probed is complementary to the higher frequencies being observed with Michelson interferometers on Earth and, ultimately, in space. Detection of a GW background due to thousands of merging galaxies in the detectable portion of the universe may be less than a decade away. The advent of the Square Kilometer Array will almost certainly lead to such a detection and the ability to characterize the spectrum and single out the few brightest sources for further study. All of this is being done with multi-purpose radio telescopes that are also being used for a wide variety of other investigations. Which will hit the gravitational wave jackpot first: a laser-powered Michelson interferometer or a Pulsar Timing Array? -

\section{About the author}

Dan Stinebring has been observing pulsars since 1976 and worked in Joe Taylor's group at Princeton from $1985-1990$. Since 1990 he has been a professor at Oberlin College. In 200607, he spent a sabbatical year at Leiden University

\section{References}

[1] J.B. Camp and N.J. Cornish, Annu. Rev. Nucl. Part. Sci. 54 (2004), 525

[2] S. Detweiler, Astrophysical Journal 234 (1979), 1100

[3] R.W. Hellings and G.S. Downs, Astrophysical Journal 265 (1983), L39

[4] R. Blandford, R. Narayan, and R.W. Romani, J., Astrophys. Astr. 5 (1984), 369

[5] F.A. Jenet, G.B. Hobbs, W. van Straten, R.N. Manchester, M. Bailes, J.P.W. Verbiest, R.T. Edwards, A.W. Hotan, J.M. Sarkissian, and S.M. Ord, Astrophysical Journal 653 (2006), 1571

[6] F.A. Jenet, G.B. Hobbs, K.J. Lee, R.N. Manchester, Astrophysical Journal 625 (2005), L123

[7] P.C.W. Davies, The search for gravity waves, Cambridge Univ. Press (1980).

[8] G.B. Hobbs, Proc. Astr. Soc. Aust 22 (2005), 179

[9] R.N. Manchester, AIP Conf. Proc. 983 (2008), 584

[10] L.P. Grishchuk, Physics-Uspekhi 48 (2005), 1235

[11] T. Damour and A. Vilenkin, Phys. Rev. D 71 (2005), 063510

[12] B.W. Stappers, M. Kramer, A.G. Lyne, N. D’Amico, and A. Jessner, Chin. J. Astron. Astrophys. 6-2 (2006), 298

[13] (NANOGrav) http://arecibo.cac.cornell.edu/arecibo-staging/nanograv/

[14] M. Kramer, New Astron. Rev. 48 (2004), 993

[15] J. M. Cordes, M. Kramer, T.J.W. Lazio, B.W. Stappers, D.C. Backer, and S. Johnston, New Astron.Rev. 48 (2004), 1413

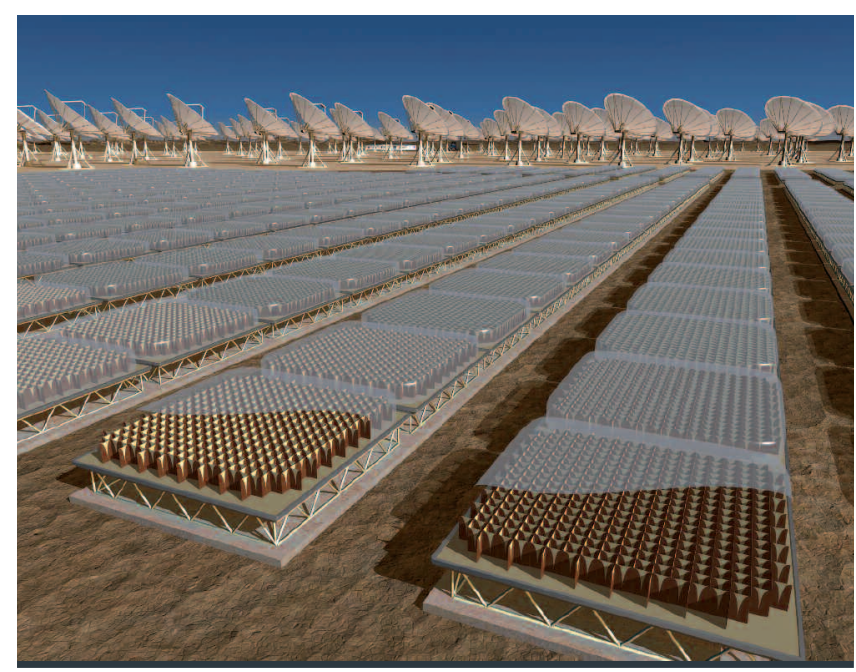

$\Delta$ FIG. 4: Artist's rendition of the central portion of the Square Kilometer Array. This fundamental instrumentation advance in astronomy will have a total collecting area of about $10^{6} \mathrm{~m}^{2}$ consisting of innovative electronic tiles and parabolic dishes, concentrated in a central core but extending in a hierarchical fashion out to many thousands of kilometers. It will be built in either western Australia or southern Africa, with construction commencing in 2011, first science in 2014, and completion around 2020. [Figure from http://www.skatelescope.org] 